\title{
What are bioplastics?
}

\section{Material types, terminology, and labels - an introduction}

\section{Bioplastics are a large family of different materials}

Bioplastics are not just one single material. Instead, they comprise of a whole family of materials with differing properties and applications. A plastic material is defined as a bioplastic if it is either bio-based, biodegradable, or features both properties.

\section{Bioplastics are bio-based, biodegradable, or both.}

Bio-based: The term 'bio-based' means that the material or product is (at least partly) derived from biomass (plants). Biomass used for bioplastics stems from e.g., corn, sugarcane, or wood.

Biodegradable: Biodegradation is a biochemical process in which microorganisms that are available in a certain environment, convert materials into water, carbon dioxide, and biomass. The process of biodegradation depends on the surrounding environmental conditions (e.g., temperature, inoculum, humidity) and on the material or the application itself.

Compostable: Compostability describes the property of being biodegradable under industrial or home composting conditions.

\section{'Bio-based' does not equal 'biodegradable'}

The property of being biodegradable does not depend on the resource basis of a material but is linked to its chemical structure. In other words, there are fully bio-based plastics that are not biodegradable, and, on the other hand, some fossil-based plastics can biodegrade under certain environmental conditions.

\section{Benefits of bioplastics}

In search of new material solutions and keeping an eye on the goal of sustainable sourcing, production and consumption, bioplastics have several advantages. The use of renewable biomass to produce bioplastics is the key for:

Increasing resource efficiency by the means of:

- The resources (crops) being cultivated on an at least annual basis;

- The principle of cascade use, as biomass can first be used for materials that can in some cases be reused and recovered, and eventually for energy recovery

Reducing the carbon footprint and greenhouse gas (GHG) emissions of materials and products

Saving fossil resources by substituting them step by step

Getting independent from fossil resources such as crude oil and therefore become independent from related exports 


\section{Material types - three main groups}

The family of bioplastics can be divided into three main groups:

1. bio-based or at least partly bio-based, non-biodegradable plastics such as bio-based polyetheylene (PE), bio-based polypropylene (PP), or bio-based polyethylene terephthalate (PET) - so-called drop-ins - and technical performance polymers, such as numerous bio-based polyamides (PA), polytrimethylene terephthalate (PTT) or totally new polymers, such as polyethylene furanoate (PEF);

2. plastics that are both bio-based and biodegradable, such as polylactic acid (PLA), and polyhydroxyalkanaotaes (PHAs), polybutylene succinate (PBS), or different starch blends;

3. plastics that are based on fossil resources but are biodegradable, such as polybutylene adipate terephthalate (PBAT), that may well be produced at least partly from bio-based feedstock in the future and in some cases already are.

The potential of bioplastics will shape the future of the plastics industry.
The 'material coordinate system of bioplastics' depicts the common types of bioplastics and how they are classified according to their biodegradability and bio-based content.

\section{Examples of established bioplastic materials}

\section{Bio-based, non-biodegradable polyolefins and polyesters}

Commodity plastics like PE, PP, and PET can also be made from renewable resources, such as sugarcane or plant oil waste. Bio-based PE is already being produced at large scale and is found in various packaging solutions. Bio-based PP can be used in the same applications as the conventional counterpart - from plastic containers to technical items. Biobased polyester PET can be used for renewable textile fibres but is predominantly found in food packaging, such as for beverage bottles. Most bio-based PET in the market is currently partially bio-based, as only the MEG (monoethylene glycol) building block is made from renewable resources. However, $100 \%$ bio-based PET can generally be produced and may be seen in the market in the future as well. These materials are also known as 'drop-in' bioplastics. The period from development to commercialisation of materials, e.g., bio-based PE or bio-based PET, is considerably shorter. A new fully bio-based polyester, PEF, is expected to enter the market very soon. It can be used for the same applications as PET but offers improved barrier properties.

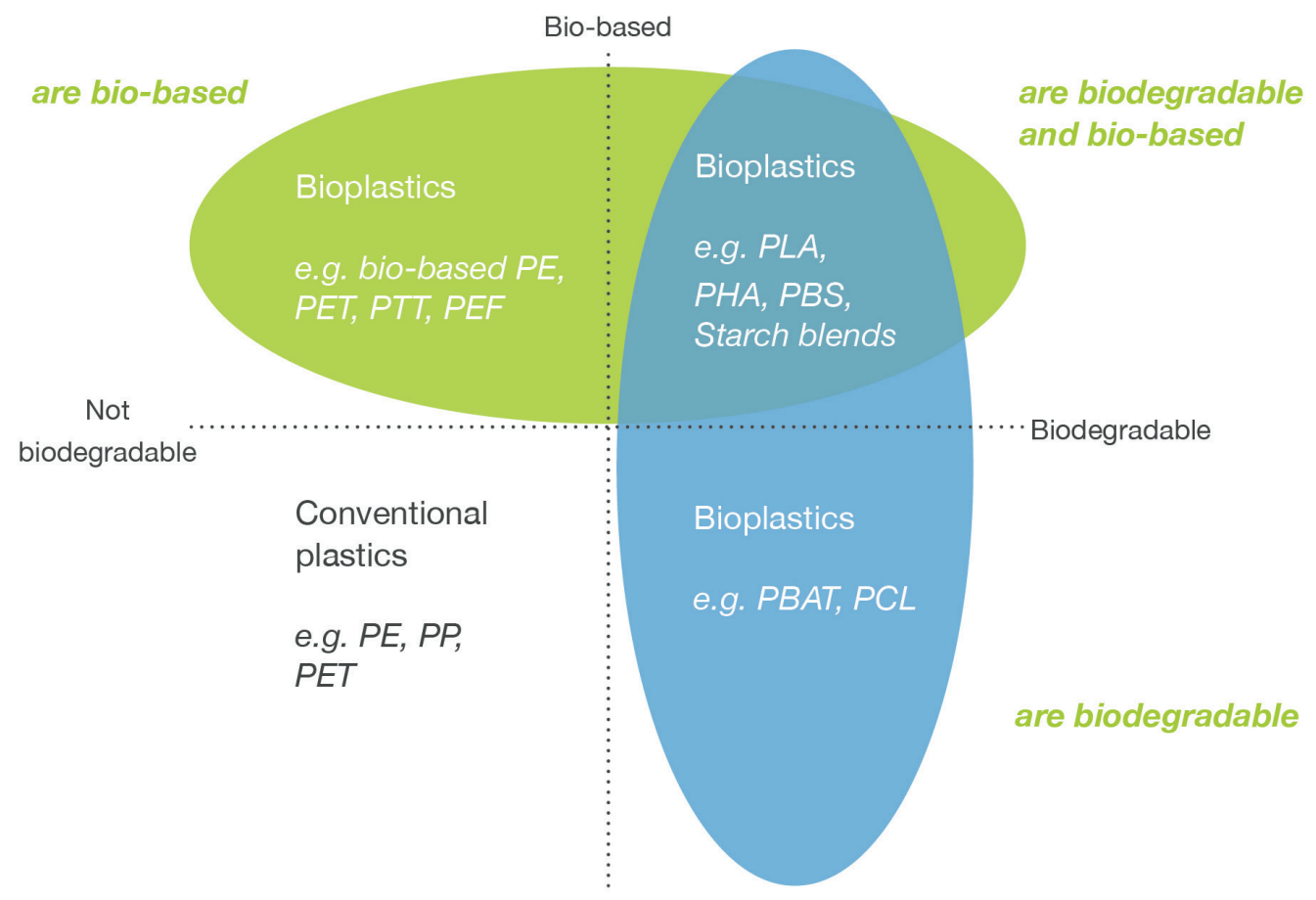

Fossil-based 
Bio-based, non-biodegradable technical/performance polymers

This large group comprises many specific polymers, such as bio-based polyamides (PA), polyesters, such as polytrimethylene terephthalate (PTT), bio-based polyurethanes (PUR) or some bio-based polyepoxides. Their use can be quite diverse. Some typical technical applications are textile fibres (seat covers, carpets), automotive applications like foams for seating, casings, cables, hoses, and covers - to name but a few. Usually, their operating life lasts several years. Therefore, they are referred to as durable bioplastics for which biodegradability is not a sought-after property. Their performance is often superior compared to conventional options.

\section{Bio-based, biodegradable plastics}

This group includes starch blends made of thermoplastic starch (TPS) and other biodegradable polymers as well as innovative polyesters, such as polylactic acid (PLA) or polyhydroxyalkanoates (PHAs). They can primarily be used for short-lived products, such as packaging', but more and more long-time applications can be seen as well. Some of these materials biodegrade predominately under composting conditions, but others also in less controlled environments.

Several materials in this group, primarily PLA, are heading for a new path - end- of-life solutions besides industrial compostability, such as mechanical but also chemical recycling. The renewable content of these materials is now the focus of attention and technical development. This dynamic development proves that bioplastics have the potential to shape the plastics industry and to produce new innovative and competitive materials.

\section{Biodegradable, fossil-based plastics}

They are a comparatively small group and are mainly used in combination with PLA or other biodegradable plastics, because they enhance the application-specific performance through improved mechanical properties. These biodegradable plastics are currently still mainly produced using fossil feedstock. However, partially bio-based versions of these materials have already been developed and will be available in the near future. Materials like PBAT and polycaprolactone belong in this category.

\section{Standards, certifications, and labels}

Below, the most relevant standards in Europe as well as corresponding independent third-party labels for bioplastics are listed ${ }^{2}$. However, the list does not reflect specific recommendations of European Bioplastics.

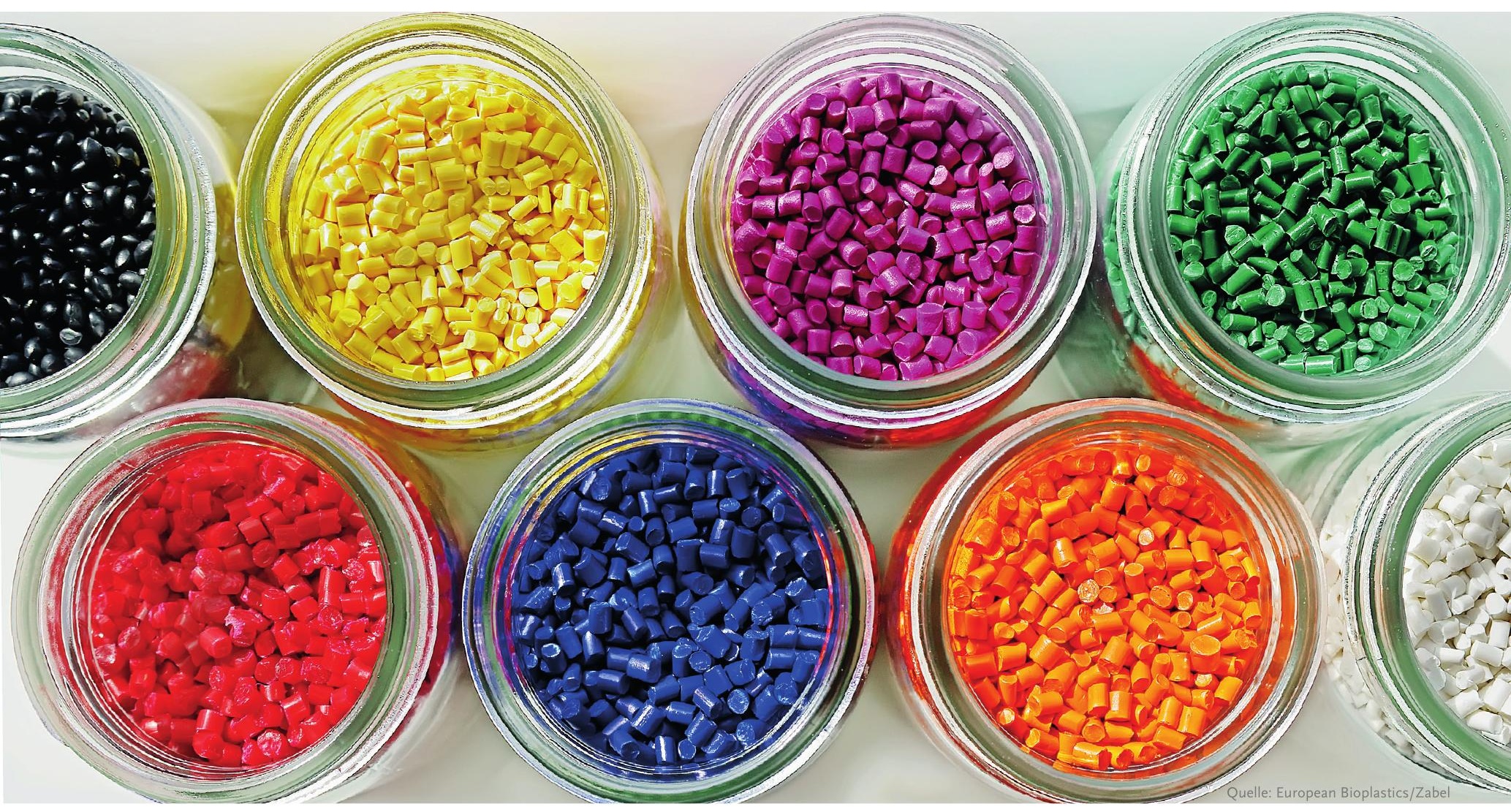




\section{Bio-based content and carbon content}

Companies producing bio-based plastics can either indicate the 'bio-based carbon content' or the 'bio-based (mass) content' of their products. As these units of measurement differ, the typical numeric percentage value will differ, too and must be taken into account, especially when making comparisons.

A well-established methodology to measure the bio-based carbon content in materials or products is the $14 \mathrm{C}$-method (EU standard EN 16640, international standard ISO 166202 corresponding US-standard: ASTM 6866). Certification schemes and derived product labels based on the European and the U.S. standard are available - for example by the Belgian certifier TÜV Austria or German certifier DIN CERTCO (OK biobased and DIN geprüft biobased).
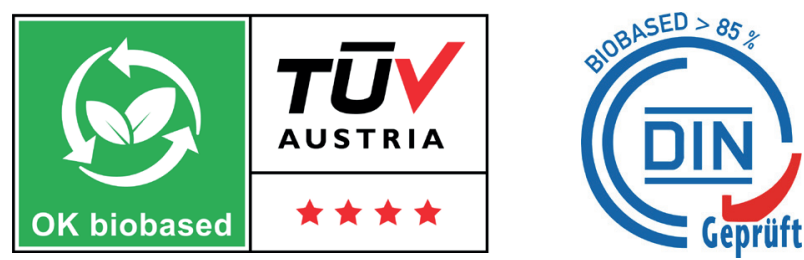

A material or product can also be specified as bio-based by indicating its bio-based (mass) content. This option is complementary to the $14 \mathrm{C}$-method and takes chemical elements other than the bio-based carbon into account, such as oxygen, nitrogen, and hydrogen. EN 16785-1 has been developed describing this method, and an according certification scheme has been published by the Netherlands Standardisation Institute (NEN). There is an equivalent ISO standard (ISO 16620-4).

\section{Biodegradability in different environments}

\section{The term 'biodegradability' is only unambiguous, if environment and time are specified.}

It is misleading to merely claim biodegradability or compostability without any standard specification. If a material or product is advertised to be biodegradable, further infor- mation about the timeframe, the level of biodegradation, and the required surrounding conditions should be provided, too.

Wherever possible, European Bioplastics recommends focussing on the more specific claim of industrial compostability $^{3}$ and to back it up with corresponding standard references (EN 13432 / EN14995, ISO 18606/ISO 17088 or ASTM D6400), a certification, and an according label (Seedling label via TÜV Austria Belgium or DIN CERTCO, OK industrial compost label via TÜV Austria Belgium).
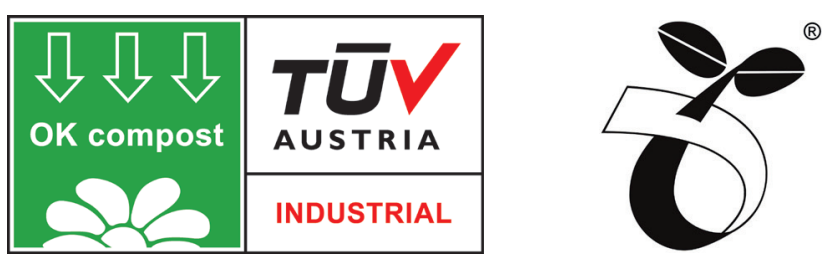

If a product is specified to be compostable, the claim is not only unambiguous (i.e., to be treated in an industrial composting plant), but there is another big benefit: It differentiates itself from products marketed to be 'oxo-biodegradable' or similar claims. Products marketed as oxo-biodegradable do not fulfil the requirements of EN 13432 on industrial compostability and have been prohibited to be sold in the EU.

Recently, there has also been growing interest to test and certify the biodegradability of materials outside of industrial composting. After several national standards and a long-established certification scheme by TÜV Austria, the standard EN 17427 has been published, providing requirements for the home compostability of carrier bags under well managed conditions. The soil-biodegradability of mulch films can be shown by referring to EN 17033. For both, home compostability and soil-biodegradability, corresponding certification schemes and labels exist.

\section{You might also be interested in the following publications:}

- Environmental Communications Guide

- Bioplastics facts and figures

- Relevant standards and labels for bioplastics

http://en.european-bioplastics.org/news/publications/
Phone: +49.3028482350

Email: info@european-bioplastics.org

Email: press@european-bioplastics.org
For more information please visit: www.european-bioplastics.org twitter.com/EUBioplastics 